\title{
Late-life falling and depressive symptoms associated with the risk of Parkinson's disease: a nationwide cohort data analysis
}

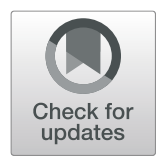

Yu Jin Jung ${ }^{1}$, Ryul Kim², Dallah Yoo ${ }^{3}$, Kyungdo $\mathrm{Han}^{4}$ and Jee-Young Lee $5^{5^{*}}$

\begin{abstract}
Background: This study aimed to evaluate the relationship between the history of late-life falling and the development of Parkinson's disease (PD) and investigate whether depressive symptoms interact with falling to increase PD risk.

Methods: We identified 1,223,726 subjects without PD who underwent the National Screening Program for Transitional Age at 66 years between 2009 and 2013 using the National Health Cohort database. In this program, every participant was assessed whether they experienced falling for the past six months. Depressive symptoms were evaluated with a three-item questionnaire extracted from the Geriatric Depression Scale. Incident PD was traced for a mean $4.23 \pm 1.50$ years. Cox proportional hazard regression models were used to assess the risk of PD by falling history with and without depressive symptoms after adjusting for other confounding variables.

Results: In this cohort, the PD incidence rate was 1.30 and 1.03 cases per 1000 person-years in groups with and without falling and 1.34 and 1.00 cases per 1000 person-years in groups with and without depressive symptoms. The predictive risk of PD was increased by either a history of falling ( $H R=1.24 ; 95 \% \mathrm{Cl} 1.11-1.40)$ or the presence of depressive symptoms ( $\mathrm{HR}=1.31 ; 95 \% \mathrm{Cl} 1.21-1.42)$ after adjusting for possible confounding variables. For individuals with both falling and depressive symptoms, PD risk increased further ( $\mathrm{HR}=1.66 ; 95 \% \mathrm{Cl} 1.40-1.97)$, but with sexdifferences. The two factors increased PD risk in a sub-additive manner in men, whereas synergistically in women.

Conclusions: This national cohort database shows that late-life depressive symptoms accompanied by a falling event pose an increase in the risk of PD in older adults.
\end{abstract}

Keywords: Falling, Depression, Parkinson's disease

\section{Background}

Parkinson's disease (PD) is one of the most common neurodegenerative disorders, and its prevalence is markedly increasing [1]. Given the growing recognition that neurodegeneration begins decades before the clinical appearance of parkinsonian motor symptoms, it becomes an important topic to identify early-stage or preclinical

\footnotetext{
* Correspondence: wieber04@snu.ac.kr

${ }^{5}$ Department of Neurology, Seoul National University-Seoul Metropolitan Government Boramae Medical Center, Seoul National University College of Medicine, 20 Boramae-ro 5-gil, Dongjak-gu, Seoul 07061, South Korea Full list of author information is available at the end of the article
}

PD [2]. The proposed clinical markers of prodromal PD include hyposmia, constipation, sleep disturbances, and mood disorders [2].

Falling is a multifactorial phenomenon and may be affected by both motor and non-motor symptoms in PD patients. Factors that increase the risk of falling in PD include a compromised gait and balance relating to parkinsonism and cognitive impairments and mood disturbances, such as depression and anxiety [3]. Depression is a common non-motor symptom of PD [4] and it frequently precedes the onset of parkinsonism in PD [5]. 
Falls and depressive symptoms are common problems in community-dwelling older adults and are asignificant burden to society [6]. Individuals with falls and depressive symptoms share several risk factors, including a slow walking speed and reaction time, poor balance, reduced muscle strength, and cognitive impairment $[7,8]$. Depressive symptoms and treatment of depression with antidepressants are associated with higher falling risk in older adults, but the relationship between depressive symptoms and falls was independent of antidepressant use and physical and cognitive factors [9]. Although depressive symptomatology was found to be consistently associated with falls in the general aged population, there have been only a few studies on the relationship between falls and depressive symptoms in PD development [10].

This study aimed to evaluate how falling events in the aged population could predict the future development of $\mathrm{PD}$ and investigate whether depression interacts with falling to increase PD risk in older adults. We used a large population-based cohort dataset from the special health screening program called the National Screening Program for Transitional Ages (NSPTA) provided by the Korean National Health Insurance (KNHI) service.

\section{Methods}

\section{Data source and study population}

The KNHI is a mandatory public health insurance system in South Korea [11], which provides a special health screening program, called the NSPTA, for people who have reached two target ages, 40- and 66 years-old [12]. In the program for the 66-year-old age group, the NSPTA includes a questionnaire designed to screen for mental health conditions and geriatric physical function [12].

We used the National Health Insurance Service-National Health Screening Cohort (NHIS-HEALS) database. The NHIS-HEALS contains demographic information such as age and sex, insurance premium (a proxy for economic status), disability status (recorded on six grades by the National Registration for Disability), the biennial National Health Screening Program (NHSP) [13] data, and information on medical service use for each individual with prescription data both at the outpatient clinic and during hospitalization under diagnostic codes following the modified version of the tenth edition of the International Classification of Diseases (ICD-10) [14]. The Korean government has implemented a benefit expansion policy for patients with PD, and this policy has compulsory registration with the governmental system at the first clinical visit with a PD diagnosis. Therefore, we identified patients with PD if individuals were both matched for the ICD-10 code of PD (G20) and registration to the Korean government's benefit expansion policy. We considered the date of the earliest claim with a PD diagnosis as the index date. Within the
NHIS-HEALS database, we identified 1,223,726 eligible individuals who had participated both in the NHSP and the NSPTA at age 66, between 2009 and 2013.

We excluded individuals who had a diagnosis of PD before $(n=4638)$ and during the 1-year lag period $(n=$ 4455) of the health screening date. Moreover, individuals who had baseline cognitive impairment, assessed by the scores of the Prescreening Korean Dementia Screening Questionnaire (KDSQ-P) $[15] \geq 4, \quad(n=163$, 069) were excluded, because cognitive impairment was known to be a common risk factor of falls and depressive symptoms $[7,8]$. Individuals with missing data for at least one variable $(n=43,372)$ were also excluded. The final study population was followed until the occurrence of PD, death, or the last follow-up date, December 31, 2017 (Fig. 1).

\section{Variables}

At the time of the NSPTA exam, all participants were asked whether they experienced any falling during the past 6 months. We collected information on the following variables: sex, smoking, alcohol consumption, exercise, economic status, body mass index (BMI), and comorbid conditions (hypertension, diabetes mellitus, and dyslipidemia), which have been considered to be related to the development of PD. Regular exercise was defined as $\geq 20 \mathrm{~min}$ of vigorous-intensity physical activity, three or more days a week, or $\geq 30$ min of moderate-intensity physical activity, five or more days a week. To screen for depressive symptoms, three questions extracted from the Geriatric Depression Scale (GDS), including loss of interest, feelings of uselessness, and feeling without hope, were used during NSPTA. If individuals answered affirmatively to any of the three questions, they were defined as having depressive symptoms in previous studies [16, 17].

\section{Statistical analysis}

Data are summarized as numbers with percentages for categorical variables and mean values with standard deviations (SD) for continuous variables. We analyzed baseline characteristics with student t-tests or chi-square tests. P-values represent statistical differences between the two groups. We used the Kaplan-Meier curve to describe the cumulative incidence of PD by time graphically and tested a statistical significance of the curves by falling or depression using a log-rank test. We also conducted multivariate analyses using a Cox proportional hazard regression model. We made adjustments for sex in model 1 and sex, smoking, alcohol consumption, exercise, and household income status in model 2 . Besides, hypertension, diabetes mellitus, and hypercholesterolemia were further adjusted in model 3. The hazard ratios (HRs) and 95\% confidence intervals (CIs) for the risk of PD in each model were estimated. The 


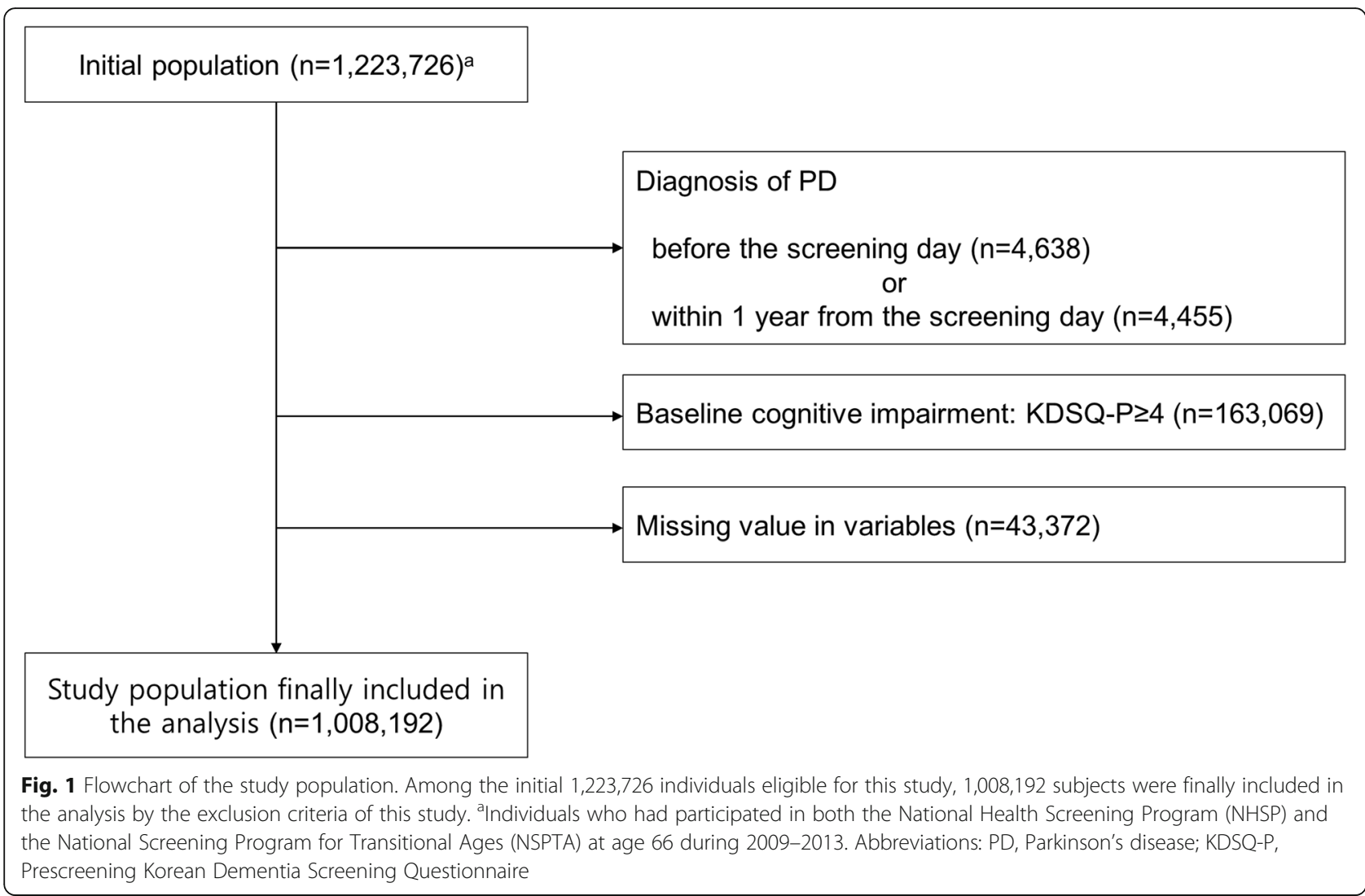

HRs for the presence of falling by the presence/absence of depressive symptoms for PD development were also assessed by multivariable Cox proportional hazards regression models. All statistical analyses were performed using SAS (version 9.4; SAS Institute Inc., Cary, NC) with the significance level set at 0.05 (two-tailed).

\section{Results}

\section{Baseline characteristics of the study population}

Finally, a total of 1,008,192 participants were included in the analysis, as described in Fig. 1. The baseline age was 66 years old. Among the 1,008,192 subjects included in the analysis, $63,939(6.7 \%)$ stated that they had fallen in the past 6 months. The baseline characteristics of the study population are summarized in Table 1 . The percentage of women was higher in the falling group compared with the no-falling group $(67.2 \%$ vs. $52.0 \%)$. Individuals with falling were more likely to be nonsmokers $(76.4 \%$ vs. $69.0 \%)$ and non-drinkers $(77.0 \%$ vs. $71.3 \%)$ and had a lower household income $(25.6 \%$ vs. 24.4\%) and a higher BMI (24.5 vs. 24.3). The falling group also consisted of a lower proportion of subjects with regular exercise (41.8\% vs. $46.8 \%)$. Hypertension (55.9\% vs. $54.2 \%)$, diabetes mellitus $(22.4 \%$ vs. $20.1 \%)$, and hypercholesterolemia $(40.6 \%$ vs. $36.4 \%)$ were more frequent in the falling group than those without a falling history. Depressive symptoms were also more prevalent in the falling group than those without a falling history (30.2\% vs. $11.4 \%)$.

\section{Parkinson's disease risk predicted by falling and depressive symptoms in late life}

In this study cohort, the mean follow-up period was $4.23 \pm 1.5$ years. The mean time to PD development was $4.23 \pm 1.5$ years in the falling group, whereas it was $4.24 \pm 1.5$ years in those without a history of falling. The incidence rates of PD were 1.30 and 1.03 cases per 1000 person-years in groups with and without falling history, respectively. Figure 2-A depicts Kaplan-Meier curves showing the relationship between the history of falling and PD incidence. PD risk was higher in the group with a history of falling than those without any falling. Unadjusted HR for PD development was $1.26(95 \% \mathrm{CI}=$ 1.13-1.41) in the falling group with reference to the non-falling group. In the adjusted models, a history of falling was also significantly associated with an increased risk of PD: adjusted $\mathrm{HR}=1.25$; $95 \% \mathrm{CI} 1.12-1.40$ in model 1, adjusted $H R=1.25 ; 95 \%$ CI $1.12-1.40$ in model 2, and adjusted $\mathrm{HR}=1.24 ; 95 \%$ CI 1.11-1.40 in model 3 (Table 2). Regarding depressive symptoms in this cohort, incidence rates of PD were 1.34 and 1.00 cases per 1000 person-years in groups with and without depressive 
Table 1 Baseline characteristics by the presence of falling history

\begin{tabular}{|c|c|c|c|c|}
\hline Variables & Total $(\boldsymbol{n}=1,008,192)$ & $\begin{array}{l}\text { Individuals with the fall } \\
\text { history }(\boldsymbol{n}=63,939)\end{array}$ & $\begin{array}{l}\text { Individuals without the fall } \\
\text { history }(\boldsymbol{n}=944,253)\end{array}$ & $\boldsymbol{P}$ value \\
\hline Age, years & 66.0 & 66.0 & 66.0 & \\
\hline Sex & & & & $<0.001$ \\
\hline Male & $474,284(47.0)$ & $20,961(32.8)$ & $453,323(48.0)$ & \\
\hline Female & $533,908(53.0)$ & $42,978(67.2)$ & $490,930(52.0)$ & \\
\hline Smoking & & & & $<0.001$ \\
\hline Never smoker & $699,442(69.4)$ & $48,737(76.4)$ & $650,705(69.0)$ & \\
\hline Ex-smoker & $175,003(17.4)$ & $8450(13.2)$ & $166,553(17.7)$ & \\
\hline Current smoker & $132,666(13.2)$ & $6642(10.4)$ & $126,024(13.4)$ & \\
\hline Alcohol $^{a}$ & & & & $<0.001$ \\
\hline Non-drinker & $719,182(71.7)$ & $48,987(77.0)$ & $670,195(71.3)$ & \\
\hline Moderate drinker & $244,632(24.3)$ & $12,504(19.7)$ & $232,128(24.7)$ & \\
\hline Heavy drinker & $39,995(4.0)$ & $2126(3.3)$ & $37,869(4.0)$ & \\
\hline Regular exercise ${ }^{b}$ & $467,788(46.5)$ & $26,687(41.8)$ & $441,101(46.8)$ & $<0.001$ \\
\hline Household income status percentiles & & & & 0.001 \\
\hline$\leq 20$ (low) & $246,929(24.5)$ & $16,388(25.6)$ & $230,541(24.4)$ & \\
\hline $30-50$ & $236,518(23.5)$ & $14,454(22.6)$ & $222,064(23.5)$ & \\
\hline $60-80$ & $317,183(31.5)$ & $20,073(31.4)$ & $297,110(31.5)$ & \\
\hline$\geq 90$ (high) & $207,562(20.5)$ & $13,024(20.4)$ & $194,538(20.6)$ & \\
\hline Body mass index, $\mathrm{kg} / \mathrm{m}^{2}$ & $24.32 \pm 3.03$ & $24.53 \pm 3.18$ & $24.31 \pm 3.02$ & $<0.001$ \\
\hline Hypertension & $546,896(54.3)$ & $35,698(55.9)$ & $511,198(54.2)$ & $<0.001$ \\
\hline Diabetes mellitus & $204,168(20.3)$ & $14,329(22.4)$ & $189,839(20.1)$ & $<0.001$ \\
\hline Hypercholesterolemia & $369,783(36.7)$ & $25,918(40.6)$ & $343,865(36.4)$ & $<0.001$ \\
\hline Depressive symptom & & & & $<0.001$ \\
\hline No & $879,380(87.4)$ & $44,523(69.8)$ & 834,857 (88.6) & \\
\hline Yes & $126,990(12.6)$ & $19,297(30.2)$ & 107,693 (11.4) & \\
\hline Follow up duration & $4.23 \pm 1.5$ & $4.24 \pm 1.5$ & $4.23 \pm 1.5$ & 0.147 \\
\hline
\end{tabular}

Data are $\mathrm{n}(\%)$ and the mean \pm standard deviation. P-values are calculated by the student $\mathrm{t}$-test for continuous variables and by the chi-square test for categorical variables

${ }^{a}$ Daily amount of alcohol consumption was categorized as follows: heavy drinker, $\geq 30 \mathrm{~g} /$ day; moderate drinker, 1-30 g/day; and nondrinker

${ }^{\mathrm{b}}$ Regular exercise indicates $\geq 20$ min of vigorous-intensity physical activity $\geq 3$ days a week or $\geq 30$ min of moderate-intensity physical activity $\geq 5$ days a week

symptoms. Depressive symptoms were significantly associated with an increased risk of PD: adjusted $\mathrm{HR}=1.31$; 95\% CI $1.21-1.42$ in model 1 , adjusted $\mathrm{HR}=1.32 ; 95 \%$ CI $1.22-1.43$ in model 2 , and adjusted $\mathrm{HR}=1.31 ; 95 \%$ CI 1.21-1.42 in model 3 (Table 2). Figure 2-B shows the Kaplan-Meier curves for the relationship between depressive symptoms and PD incidence.

Interactive impact of falling and depressive symptoms on the risk of Parkinson's disease

As summarized in Table 3, falling in the presence of depressive symptoms seemed likely to increase the PD risk synergistically in the whole study population $(\mathrm{HR}=$ 1.66; 95\% CI 1.40-1.97). However, when we analyzed both sexes separately, the impacts of falling and depressive symptoms were different. In men, the risk of PD increased even if either depressive symptoms or history of a falling was present $(\mathrm{HR}=1.28 ; 95 \% \mathrm{CI} 1.11-1.47, \mathrm{HR}=$ $1.41 ; 95 \%$ CI 1.14-1.76) and increased further when both factors coexisted $(\mathrm{HR}=1.60 ; 95 \% \mathrm{CI} 1.17-2.20)$ in a sub-additive manner. On the other hand, in women, a history of falling alone did not increase the risk of PD $(\mathrm{HR}=0.97 ; \quad 95 \%$ CI 0.81-1.16), whereas depressive symptoms alone increased the PD risk $(\mathrm{HR}=1.25 ; 95 \%$ CI 1.12-1.40). The risk of PD increased by $67 \%$ in the presence of both a history of falling and depressive symptoms $(\mathrm{HR}=1.67 ; 95 \%$ CI $1.36-2.04)$ compared to those with double negative symptoms. Depressive symptoms with a falling history synergistically increased the risk of PD in women. (Table 3, Fig. 3).

\section{Discussion}

This national cohort data analysis shows that recent falling accompanied by depressive symptoms in the aged 


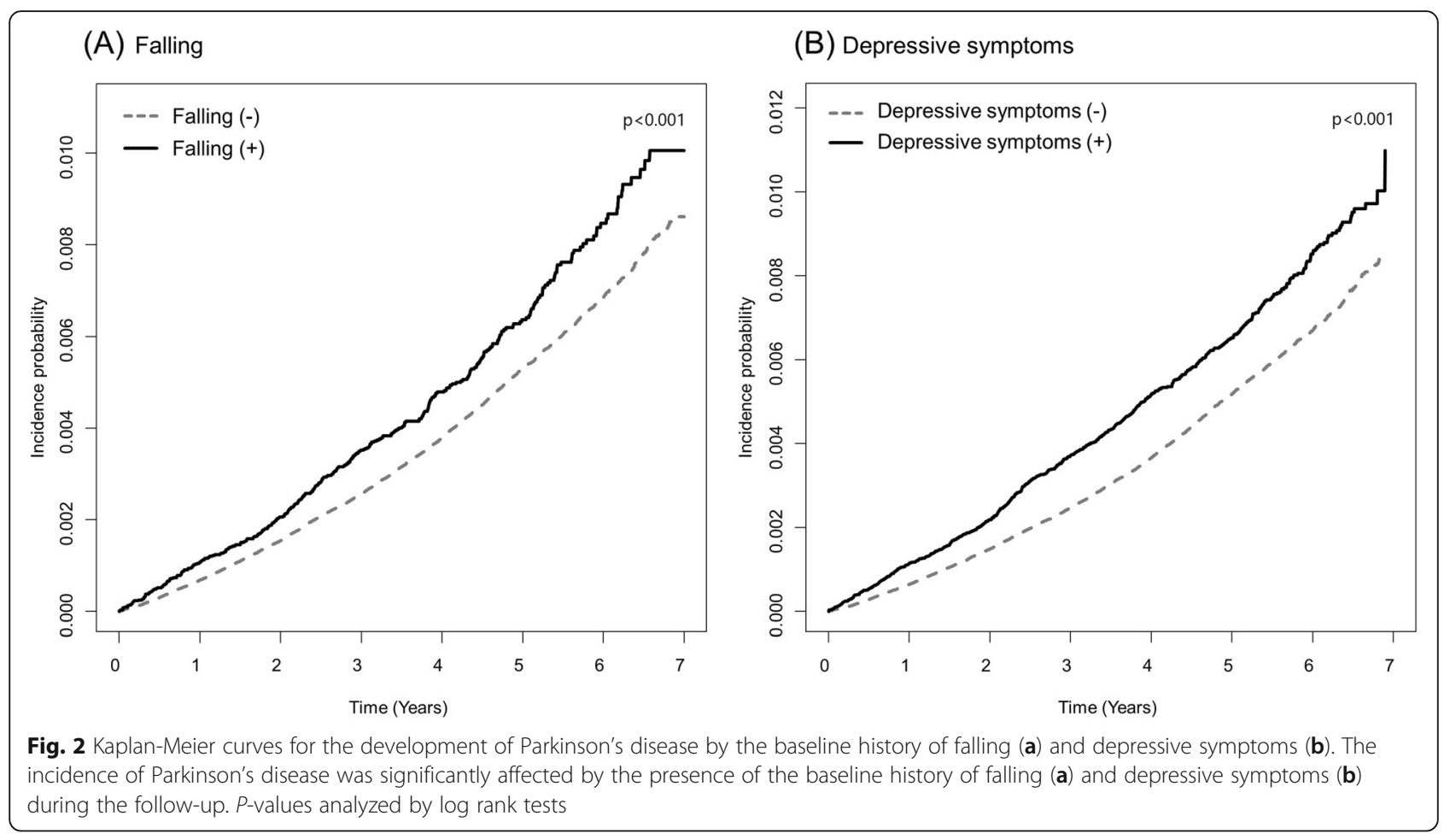

population is associated with an increased risk of PD by $66 \%$. One nationwide cohort study reported that $18 \%$ of PD patients, compared with $11.5 \%$ of controls, had experienced injurious falls in the 20 years before the diagnosis of PD [18] and a recent meta-analysis found that depression was associated with a 2.2-fold increase in the incidence of PD [19]. However, to the best of our knowledge, this is the first study revealing the impact of falling in combination with depressive symptoms in older adults' future PD development.

The present study demonstrated an appreciably lower percentage $(6.7 \%)$ of falls in the past six months than that $(27 \%)$ of the twelve-month prevalence of falls in a recent meta-analysis, and the falling was even more prevalent in older ages [20]. The estimated pooled prevalence of depression was $34.4 \%$ in India from a meta-analysis [21], whereas our findings revealed a relatively lower incidence (12.6\%) of depressive symptoms in our population. The discrepancies appeared to be due to the difference in the age and characteristics of the included subjects. Since this study was conducted on relatively young senior citizens of a single age of 66 years and based on the health screening program, the data might be more likely to reflect a healthy and active aged population than other studies.

Falling and depressive symptoms may be a sign of disconnection in the fronto-striato-limbic circuit that can be a preclinical functional change in the brain related to PD. Depression is part of the 'emotional status' and the amygdala is an essential neural structure of the limbic network that plays an integral role in emotional processing [22]. The amygdala and associated limbic structures have extensive efferent connections to areas involved in posture, including vestibular nuclei, the reticular formation and

Table 2 Incidence of PD according to the baseline fall history and depressive symptoms

\begin{tabular}{|c|c|c|c|c|c|c|c|}
\hline & \multirow{2}{*}{$\begin{array}{l}\text { Total } \\
\text { number }\end{array}$} & \multirow{2}{*}{$\begin{array}{l}\text { Number of } \\
\text { PD } \\
\text { occurrence }\end{array}$} & \multirow{2}{*}{$\begin{array}{l}\text { Person- } \\
\text { years }\end{array}$} & \multirow{2}{*}{$\begin{array}{l}\text { Incidence } \\
\text { Rate }^{a}(1 / \\
1000)\end{array}$} & \multicolumn{3}{|c|}{ Adjusted $\mathrm{HR}^{\mathrm{b}}(95 \% \mathrm{Cl})$} \\
\hline & & & & & Model 1 & Model 2 & Model 3 \\
\hline Individuals without the fall history & 944,253 & 4131 & $3,993,223.44$ & 1.03 & 1.00 & 1.00 & 1.00 \\
\hline Individuals with the fall history & 63,939 & 352 & $270,964.24$ & 1.30 & $1.25(1.12-1.40)$ & $1.25(1.12-1.40)$ & $1.24(1.11-1.38)$ \\
\hline Individuals without depressive symptoms & 879,380 & 3735 & $3,703,887.61$ & 1.00 & & & \\
\hline Individuals with depressive symptoms & 126,990 & 736 & $549,052.95$ & 1.34 & $1.31(1.21-1.42)$ & $1.32(1.22-1.43)$ & $1.31(1.21-1.42)$ \\
\hline
\end{tabular}

${ }^{a}$ Incidence rates were expressed as per 1000 person-years

${ }^{b}$ HRs were adjusted for sex (model 1), for sex, smoking, alcohol consumption, regular exercise, household income status (model 2) and for the same variables plus body mass index and the presence of hypertension, diabetes mellitus and hypercholesterolemia (model 3)

Abbreviations: $C$ confidence interval, HR hazard ratio 
Table 3 PD risk predicted by the combination of falling history and depressive symptoms

\begin{tabular}{|c|c|c|c|c|c|}
\hline & Total number & Number of PD occurrence & Person-years & Incidence Rate ${ }^{a}(1 / 1000)$ & Adjusted HR ${ }^{\mathrm{b}}(95 \% \mathrm{Cl})$ \\
\hline \multicolumn{6}{|l|}{ Total } \\
\hline Falling (-) Depression (-) & 834,857 & 3522 & $3,515,446.76$ & 1.002 & 1.00 \\
\hline Falling (-) Depression (+) & 107,693 & 598 & $467,281.91$ & 1.280 & $1.27(1.16-1.38)$ \\
\hline Falling (+) Depression (-) & 44,523 & 213 & $188,440.86$ & 1.130 & $1.11(0.97-1.28)$ \\
\hline Falling (+) Depression (+) & 19,297 & 138 & $81,771.04$ & 1.688 & $1.66(1.40-1.97)$ \\
\hline \multicolumn{6}{|l|}{ Men } \\
\hline Falling (-) Depression (-) & 409,661 & 1657 & $1,706,433.44$ & 0.971 & 1.00 \\
\hline Falling (-) Depression (+) & 42,743 & 227 & $183,166.81$ & 1.239 & $1.28(1.11-1.47)$ \\
\hline Falling (+) Depression (-) & 14,777 & 86 & $62,030.75$ & 1.386 & $1.41(1.14-1.76)$ \\
\hline Falling (+) Depression (+) & 6136 & 40 & $25,498.32$ & 1.569 & $1.60(1.17-2.20)$ \\
\hline \multicolumn{6}{|l|}{ Women } \\
\hline Falling (-) Depression (-) & 425,196 & 1865 & $1,809,013.32$ & 1.031 & 1.00 \\
\hline Falling (-) Depression (+) & 64,950 & 371 & $284,115.10$ & 1.306 & $1.25(1.12-1.40)$ \\
\hline Falling (+) Depression (-) & 29,746 & 127 & $126,410.11$ & 1.005 & $0.97(0.81-1.16)$ \\
\hline Falling (+) Depression (+) & 13,161 & 98 & $56,272.72$ & 1.742 & $1.67(1.36-2.04)$ \\
\hline
\end{tabular}

${ }^{a}$ Incidence rates were expressed as per 1000 person-years. ${ }^{b} \mathrm{HRs}$ were adjusted for sex, smoking, alcohol consumption, regular exercise, household income status, body mass index and the presence of hypertension, diabetes mellitus and hypercholesterolemia Abbreviations: $\mathrm{Cl}$ confidence interval, $\mathrm{HR}$ hazard ratio

nuclei within the basal ganglia and nucleus accumbens [23]. Because the amygdala is one of the structures with the earliest pathological involvement of PD [24], the disruption of these amygdala-related connections in depressed older adults can hypothetically cause falling before the appearance of parkinsonian motor symptoms. Furthermore, there has been increasing evidence of a relationship between limbic dysfunction and sudden gait ignition failure or freezing in PD [25]. A functional MRI study has shown that dysfunctional fronto-striato-limbic circuitry involving emotional processing could be related to gait freezing episodes in PD patients [26]. The close association between the emotional components and disrupted gait and balance supports our finding that the combination of falling and depressive symptoms is associated with an increased risk of PD development in older adults.

The concept of "Fear of falling (FOF)" could be suggested as one possible mechanism for the interactive impact of falling and depression in the aged population. Previous falls usually play a role in developing FOF [27]. However, FOF is also commonly reported in older adults who have not experienced a real fall [28], and depression is highly associated with FOF $[29,30]$. In PD, depression is a significant non-motor contributor of FOF independent of the history of real fall [10]. Therefore, depression, FOF, and falling are considerably interconnected, and they may be a sign of an increased risk of PD although the causal relationship linking depression, FOF, and the resultant falling has been unrevealed. In our cohort database, the percentage of women was higher in the falling group, and a history of falling alone did not increase the
PD development, whereas the combination of falling and depressive symptoms increased PD risk in women even higher than in men. These results were in line with several other studies that the prevalence of FOF was higher in women than in men [31] and women with apathy experienced more fall incidents than men with apathy [32], thereby suggesting the possibility of "FOF" as a candidate risk factor regardless of the history of falling as predicting the risk of PD in women, which is worth to be investigated by future studies.

Our study's strengths include the use of a homogenous sample of the Korean aged population at the same age (66 years old) and a significant follow-up period. In addition, we adjusted for potential confounders extensively in the multivariate analysis. Nevertheless, the present study has some limitations. Because this was a big data analysis, we could only track the onset time of PD by medical records, and there may be a discrepancy in the actual onset time of PD. However, we excluded individuals who had a diagnosis of PD during the 1-year lag period from the date of health screening to rule out the case in which PD has already developed. The results did not significantly vary when we analyzed the data even without the 1-year lag period. Therefore, it is less likely that our data were affected by misdiagnoses. Second, we could not include some potential confounders for PD risk in multivariate analyses. These include physical performance status, genetic causes, sleep problems and caffeine consumption. Moreover, there was a lack of information about comorbidities except for hypertension, diabetes mellitus and hypercholesterolemia and 
(A) Men

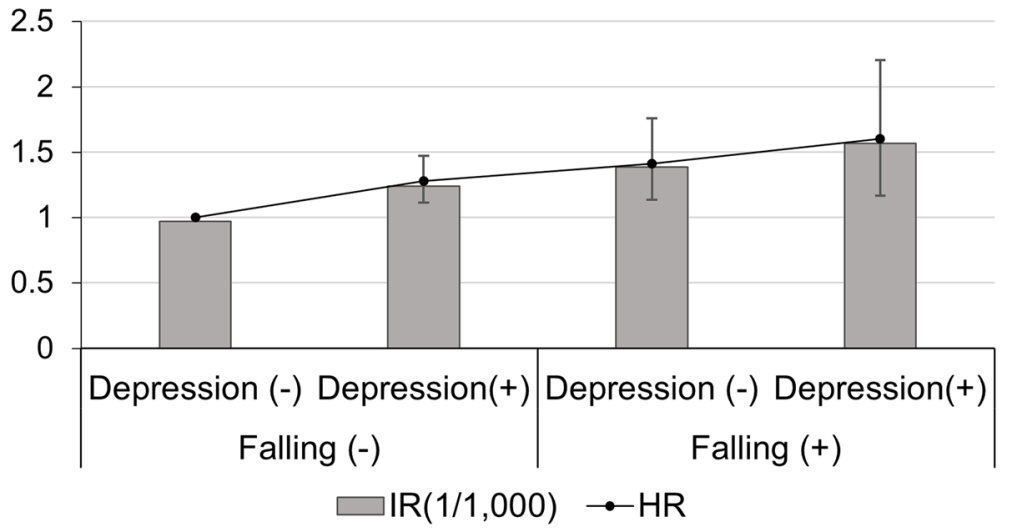

(B) Women

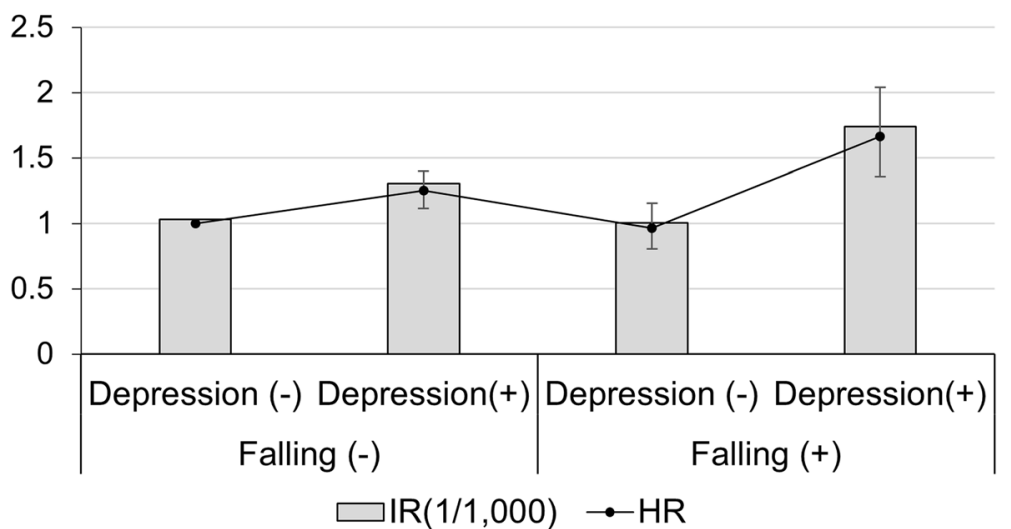

Fig. 3 The risk of Parkinson's disease predicted by the presence of both depressive symptoms and a history of falling at baseline in men (a) and women (b). In men (a), depressive symptoms and a history of falling increased the risk of PD in a sub-additive manner whereas in women (b), the risk of PD was synergistically increased by the presence of both a history of falling and depressive symptoms. Abbreviations: IR, incidence rate; $H R$, hazard ratio

medications, which could directly affect the falling and the depressive symptoms. However, this study used nationwide big data to have enough power to avoid possible risk factor stratification. Finally, assessment of falling and depressive symptoms based on a self-reported questionnaire might be affected by recall bias.

\section{Conclusion}

A history of recent falls accompanied by depressive symptoms is associated with a substantial increase in the risk of future PD development. The two factors increased PD risk in a sub-additive manner in men, whereas synergistically in women. Older adults who have both depressive symptoms and a history of recent falls may require a careful follow-up.

\section{Abbreviations}

PD: Parkinson's disease; NSPTA: National Screening Program for Transitional Ages; KNHI: Korean National Health Insurance; NHIS-HEALS: National Health Insurance Service-National Health Screening Cohort; NHSP: National Health
Screening Program; ICD: International Classification of Diseases; KDSQP: Prescreening Korean Dementia Screening Questionnaire; BMI: body mass index; GDS: Geriatric Depression Scale; FOF: Fear of falling

\section{Acknowledgements}

We thank the KNHIS for providing access to their data (No. NHIS-2019-1-051).

\section{Authors' contributions}

YJJ and JYL conceptualized and designed the survey. YJJ performed the statistical analysis, interpreted the data, drafted the manuscript. KDH performed the statistical analysis and interpreted the data. RK, DY and JYL interpreted the data and critically revised the manuscript. All authors gave approval for the final version of the manuscript and agree to be accountable for all aspects of the work.

\section{Funding}

This research received no specific grant from any funding agency in the public, commercial, or not-for-profit sectors.

\section{Availability of data and materials}

Data is available from the Korea National Health Insurance Sharing Service Institutional Data Access / Ethics Committee (https://nhiss.nhis.or.kr/bd/ay/ bdaya001iv.do) for researchers who meet the criteria for access to confidential data. Researchers can apply for the National health Insurance data sharing service upon approval by the Institutional Review Board of their 
institution. After a review by the Korea National Health Insurance Sharing Service Institutional Data Access / Ethics Committee, authors are required to pay a data access fee and confirm that other researchers will be able to access the data in the same manner as the authors.

\section{Ethics approval and consent to participate}

This study was approved by the Institutional Review Board (IRB) of Seoul National University Boramae Hospital (IRB No. 07-2018-14/062). Informed consent was waived by the IRB because the KNHIS dataset was constructed after anonymization, based on the strict confidentiality guidelines of the Korean Government

\section{Consent for publication}

Not applicable.

\section{Competing interests}

The authors declare that they have no competing interests.

\section{Author details}

'Department of Neurology, Daejeon St. Mary's Hospital, College of Medicine, The Catholic University of Korea, Seoul, Republic of Korea. ${ }^{2}$ Department of Neurology, Inha University Hospital, Incheon, Republic of Korea. ${ }^{3}$ Department of Neurology, Kyung-Hee University Hospital, College of Medicine, Kyung-Hee University, Seoul, Republic of Korea. ${ }^{4}$ Department of Statistics and Actuarial Science, Soongsil University, Seoul, Republic of Korea. ${ }^{5}$ Department of Neurology, Seoul National University-Seoul Metropolitan Government Boramae Medical Center, Seoul National University College of Medicine, 20 Boramae-ro 5-gil, Dongjak-gu, Seoul 07061, South Korea.

Received: 21 January 2020 Accepted: 4 August 2020 Published online: 10 August 2020

\section{References}

1. Calabrese VP. Projected number of people with Parkinson disease in the most populous nations, 2005 through 2030. Neurology. 2007;69(2):223-4 author reply 224.

2. Mantri S, Morley JF, Siderowf AD. The importance of preclinical diagnostics in Parkinson disease. Parkinsonism Relat Disord. 2019;64:20-28.

3. Robinson K, Dennison A, Roalf D, Noorigian J, Cianci H, Bunting-Perry L, Moberg P, Kleiner-Fisman G, Martine R, Duda J, et al. Falling risk factors in Parkinson's disease. NeuroRehabilitation. 2005;20(3):169-82.

4. Ghaddar A, Fawaz M, Khazen G, Abdallah J, Milane A. Prevalence of depression in Parkinson's disease in a Lebanese tertiary clinic. J Clin Exp Neuropsychol. 2016;38(1):51-8.

5. Lemke MR. Depressive symptoms in Parkinson's disease. Eur J Neurol. 2008; 15(Suppl 1):21-5.

6. Verma SK, Willetts JL, Corns HL, Marucci-Wellman HR, Lombardi DA, Courtney TK. Falls and fall-related injuries among community-dwelling adults in the United States. PLoS One. 2016;11(3):e0150939.

7. Caligiuri MP, Ellwanger J. Motor and cognitive aspects of motor retardation in depression. J Affect Disord. 2000;57(1-3):83-93.

8. Lord SR, Clark RD, Webster IW. Physiological factors associated with falls in an elderly population. J Am Geriatr Soc. 1991;39(12):1194-200.

9. Kvelde T, Lord SR, Close JC, Reppermund S, Kochan NA, Sachdev P, Brodaty $H$, Delbaere K. Depressive symptoms increase fall risk in older people, independent of antidepressant use, and reduced executive and physical functioning. Arch Gerontol Geriatr. 2015;60(1):190-5.

10. Franzen $\mathrm{E}$, Conradsson D, Hagstromer M, Nilsson MH. Depressive symptoms associated with concerns about falling in Parkinson's disease. Brain Behav. 2016;6(10):e00524

11. Song YJ. The South Korean Health Care System. JMAJ. 2009;52(3):206-9.

12. Kim HS, Shin DW, Lee WC, Kim YT, Cho B. National screening program for transitional ages in Korea: a new screening for strengthening primary prevention and follow-up care. J Korean Med Sci. 2012;27(Suppl):S70-5.

13. Shin DW, Cho B, Guallar E. Korean national health insurance database. JAMA Intern Med. 2016;176(1):138.

14. Lee SH, Lee SJ, Kim YJ. Region-Based Analysis of Prevalence and Incidence of Parkinson's Disease: Analysis of the National Sample Cohort in South Korea. J Clin Neurol (Seoul, Korea). 2018;14(4):478-86.

15. Jeon Y, Yun K, Kim Y. Validation of KDSQ-P as selecting elderly for KDSQ-C. Korean J Health Promot. 2010;10(2):45-52.
16. Bae JN, Cho MJ. Development of the Korean version of the geriatric depression scale and its short form among elderly psychiatric patients. J Psychosom Res. 2004;57(3):297-305.

17. Lee JE, Shin DW, Jeong SM, Son KY, Cho B, Yoon JL, Park BJ, Kwon IS, Lee J, Kim S. Association between timed up and go test and future dementia onset. J Gerontol A Biol Sci Med Sci. 2018;73(9):1238-43.

18. Nystrom $\mathrm{H}$, Nordstrom A, Nordstrom P. Risk of injurious fall and hip fracture up to 26 y before the diagnosis of Parkinson disease: nested case-control studies in a Nationwide cohort. PLoS Med. 2016;13(2):e1001954.

19. Wang S, Mao S, Xiang D, Fang C. Association between depression and the subsequent risk of Parkinson's disease: a meta-analysis. Prog NeuroPsychopharmacol Biol Psychiatry. 2018;86:186-92.

20. Elias Filho J, Borel WP, Diz JBM, Barbosa AWC, Britto RR, Felício DC. Prevalence of falls and associated factors in community-dwelling older Brazilians: a systematic review and meta-analysis. Cadernos de saude publica. 2019;35(8):e00115718.

21. Pilania M, Yadav V, Bairwa M, Behera P, Gupta SD, Khurana H, Mohan V, Baniya G, Poongothai S. Prevalence of depression among the elderly (60 years and above) population in India, 1997-2016: a systematic review and meta-analysis. BMC Public Health. 2019;19(1):832

22. Vriend C, Boedhoe PS, Rutten S, Berendse HW, van der Werf YD, van den Heuvel OA. A smaller amygdala is associated with anxiety in Parkinson's disease: a combined FreeSurfer-VBM study. J Neurol Neurosurg Psychiatry. 2016;87(5):493-500.

23. Cardinal RN, Parkinson JA, Hall J, Everitt BJ. Emotion and motivation: the role of the amygdala, ventral striatum, and prefrontal cortex. Neurosci Biobehav Rev. 2002;26(3):321-52.

24. Braak H, Del Tredici K, Rub U, de Vos RA, Jansen Steur EN, Braak E. Staging of brain pathology related to sporadic Parkinson's disease. Neurobiol Aging. 2003;24(2):197-211.

25. Ehgoetz Martens KA, Ellard CG, Almeida QJ. Does anxiety cause freezing of gait in Parkinson's disease? PLoS One. 2014;9(9):e106561.

26. Gilat M, Ehgoetz Martens KA, Miranda-Dominguez O, Arpan I, Shine JM, Mancini M, Fair DA, Lewis SJG, Horak FB. Dysfunctional limbic circuitry underlying freezing of gait in Parkinson's disease. Neuroscience. 2018;374: 119-32.

27. Kader M, Iwarsson S, Odin P, Nilsson MH. Fall-related activity avoidance in relation to a history of falls or near falls, fear of falling and disease severity in people with Parkinson's disease. BMC Neurol. 2016;16:84.

28. Makino K, Makizako H, Doi T, Tsutsumimoto K, Hotta R, Nakakubo S, Suzuki $\mathrm{T}$, Shimada $\mathrm{H}$. Impact of fear of falling and fall history on disability incidence among older adults: prospective cohort study. Int J Geriatric Psychiatry. 2018;33(4):658-62.

29. Sharaf AY, Ibrahim HS. Physical and psychosocial correlates of fear of falling: among older adults in assisted living facilities. J Gerontol Nurs. 2008;34(12):27-35.

30. Shin KR, Kang Y, Kim MY, Jung D, Kim JS, Hong CM, Yun ES, Ma RW. Impact of depression and activities of daily living on the fear of falling in Korean community-dwelling elderly. Nurs Health Sci. 2010;12(4):493-8.

31. Tomita Y, Arima K, Tsujimoto R, Kawashiri SY, Nishimura T, Mizukami S, Okabe T, Tanaka N, Honda Y, Izutsu K, et al. Prevalence of fear of falling and associated factors among Japanese community-dwelling older adults. Medicine. 2018;97(4):e9721.

32. Henstra MJ, Rhebergen D, Stek ML, Swart KMA, van Dijk SC, Zillikens MC, Oliai Araghi S, de Groot L, van Schoor NM, van der Velde N. The association between apathy, decline in physical performance, and falls in older persons. Aging Clin Exp Res. 2019;31(10):1491-9.

\section{Publisher's Note}

Springer Nature remains neutral with regard to jurisdictional claims in published maps and institutional affiliations. 